\title{
Tempos de pandemia COVID-19: sintomatologia depressiva em idosos
}

\author{
COVID-19 pandemic times: depressive symptomatology in the elderly \\ Tiempos de pandemia de COVID-19: sintomatología depresiva en los ancianos
}

Recebido: 25/10/2021 | Revisado: 04/11/2021 | Aceito: 08/11/2021 | Publicado: 13/11/2021

\author{
Marília de Freitas Lima \\ ORCID: https://orcid.org/0000-0002-8839-757X \\ Universidade Estadual da Paraíba, Brasil \\ E-mail: marilialimapsi@gmail.com \\ Edwirde Luiz Silva Camêlo \\ ORCID: https://orcid.org/0000-0003-3686-927X \\ Universidade Estadual da Paraíba, Brasil \\ E-mail: edwirde@uepb.edu.br
}

\begin{abstract}
Resumo
Introdução: Atualmente a população mundial continua enfrentando a Pandemia da COVID-19. O distanciamento social, assim como, a possibilidade de adoecer, pode acarretar novos sofrimentos para as pessoas, como a depressão, em especial para os idosos. Objetivo: Avaliar a sintomatologia depressiva de idosos em tempo de pandemia da COVID-19. Metodologia: Esta pesquisa se caracteriza como sendo transversal, descritiva e exploratória, com abordagem quantitativa. A coleta de dados foi realizada de forma online por meio de um formulário, que continha os seguintes instrumentos: Questionário sociodemográfico e clínico e a Escala de Depressão Geriátrica - EDG. Participaram, de forma não probabilística por conveniência, 44 pessoas idosas com idades variando de 60 a 87 anos. Em relação a análise de dados, foi elaborado um banco de dados com prévia codificação das respostas, usando o software Statistical Package for Social Science (SPSS) for Windows, versão 20 e programa R 3.5.1. Resultado: O perfil sociodemográfico dos participantes foi caracterizado, em sua maioria, por pessoas idosas do sexo feminino e que se declararam católicas. Ademais, a maioria dos participantes eram nordestinos, aposentados, casados, possuem baixo nível de escolaridade e renda mensal familiar entre 1 e 2 salários mínimos. Constatou-se que em 75\% idosos não há presença de depressão, enquanto $25 \%$ apresentaram sintomatologia depressiva moderada ou severa. Considerações finais: Diante disso, é possível compreender que o isolamento e/ou distanciamento social, embora necessário durante a pandemia da COVID-19, pode ocasionar efeitos prejudiciais à saúde mental da pessoa idosa.
\end{abstract}

Palavras-chave: Idosos; Pandemia; COVID-19; Depressão.

\begin{abstract}
Introduction: Currently, the world population continues to face the Pandemic of COVID-19. The social distancing and the possibility of getting sick can bring new suffering to people, such as depression, especially for the elderly. Objective: To evaluate the depressive symptoms of older people during the COVID-19 pandemic. Methodology: This research is characterized as being transversal, descriptive and exploratory, with a quantitative approach. Data collection was performed online through a form, which contained the following instruments: sociodemographic and clinical questionnaire and the Geriatric Depression Scale - GDS. Forty-four elderly people with ages ranging from 60 to 87 years participated in a non-probabilistic way for convenience. Regarding data analysis, a database was created with the previous coding of responses, using the Statistical Package for Social Science (SPSS) software for Windows, version 20, and $\mathrm{R}$ program version 3.5.1. Results: The sociodemographic profile of the participants was mainly composed of elderly females who declared themselves Catholic. Furthermore, most participants were from the Northeast, retired, married, with a low level of education and monthly family income between 1 and 2 minimum wages. We found that $75 \%$ of the elderly did not have depression, while $25 \%$ had moderate or severe depressive symptoms. Concluding remarks: Given this, it is possible to understand that isolation and social distancing, although necessary during the COVID-19 pandemic, can have harmful effects on the mental health of the elderly.
\end{abstract}

Keywords: Elderly; Pandemic; COVID-19; Depression.

\section{Resumen}

Resumen: Introducción: Actualmente, la población mundial continúa enfrentando la pandemia COVID-19. El distanciamiento social, así como la posibilidad de enfermarse, puede generar nuevos sufrimientos para las personas, como la depresión, especialmente para las personas mayores. Objetivo: Evaluar los síntomas depresivos de los ancianos durante la pandemia de COVID-19. Metodología: Esta investigación se caracteriza por ser transversal, descriptiva y exploratoria, con un enfoque cuantitativo. La recolección de datos se realizó en línea a través de un formulario, que contenía los siguientes instrumentos: cuestionario sociodemográfico y clínico y la Escala de Depresión Geriátrica - EDG. Participaron 44 personas mayores de entre 60 y 87 años de forma no probabilística por 
conveniencia. En relación al análisis de datos, se creó una base de datos con codificación previa de respuestas, utilizando el software Statistical Package for Social Science (SPSS) para Windows, versión 20 y el programa R 3.5.1. Resultado: El perfil sociodemográfico de los participantes se caracterizó principalmente por mujeres ancianas que se declararon católicas. Además, la mayoría de los participantes eran del Nordeste, jubilados, casados, con bajo nivel educativo y con ingresos familiares mensuales entre 1 y 2 salarios mínimos. Se encontró que en el $75 \%$ de los ancianos no existe depresión, mientras que el $25 \%$ presenta síntomas depresivos moderados o severos. Consideraciones finales: Ante esto, es posible entender que el aislamiento y/o el distanciamiento social, aunque necesarios durante la pandemia de COVID-19, pueden tener efectos nocivos sobre la salud mental de las personas mayores.

Palabras clave: Ancianos; Pandemia; COVID-19; Depresión.

\section{Introdução}

A mudança do perfil epidemiológico e demográfico possibilitou um dos acontecimentos sociais mais relevantes do século passado e que segue durante o século atual: o aumento da expectativa de vida das pessoas, resultando no aumento da população idosa e, logo, no envelhecimento progressivo da sociedade. Inúmeras são as razões que podem elucidar porque a expectativa de vida humana tem crescido, como por exemplo, a melhoria nas condições de saúde e os avanços da medicina, resultantes, em grande parte, das transformações sociais que ocorreram após a Revolução Industrial e as duas Grandes Guerras (Chaimowicz, 2013).

No Brasil, conforme dados do Instituto Brasileiro de Geografia e Estatística (IBGE, 2018), entre os anos de 2012 e 2017, houve um crescimento de $18 \%$ na população acima de 60 anos, equivalente a um total de 4,8 milhões de novos idosos, e totalizando uma população de aproximadamente 30,2 milhões de idosos. O IBGE (2019) também afirma, em sua Projeção da População informada em 2018, que em 2043, um quarto da população deverá possuir mais de 60 anos. Com o propósito de que os idosos tenham qualidade de vida, é necessário garantir direitos em esferas como saúde, assistência social, trabalho, dentre outros, para isso no país tais direitos são regulamentados pela Política Nacional do Idoso e o Estatuto do Idoso.

O envelhecimento é caracterizado por alterações morfológicas, psicológicas, físicas, bioquímicas e fisiológicas (Coelho, Gobbi, Costa \& Gobbi, 2013). Tais modificações indicam uma maior vulnerabilidade a enfermidades, como a depressão, podendo influenciar na qualidade de vida do idoso. Contudo, depressão não é uma consequência natural do envelhecimento, consistindo em uma morbidade psíquica relacionada a um intenso sofrimento, podendo acarretar no declínio cognitivo e maiores índices de mortalidade (Garcia, Rodrigues \& Dos Santos, 2012).

O fato da população idosa está aumentando resulta na imprescindibilidade de que se desenvolvam planos de ação para a atenção essencial e adequada no campo da saúde e social, que haja garantias de uma atendimento psicológico e médico de qualidade, assim como, uma sociedade que respeite. Durante a velhice alguns eventos negativos, graduados por idade e não normativos, podem tornar-se predominantes, tais como o falecimento de amigos e familiares, como o (a) cônjuge, perda de status social, insegurança econômica, bem como o declínio da funcionalidade física e da saúde (Fortes, Portuguez, \& Argimon, 2009).

Atualmente uma das adversidades enfrentadas pela população mundial e tida como uma das maiores emergências de saúde pública do mundo é o novo Coronavírus (COVID-19). Em dezembro de 2019 foi registrado o primeiro caso de infecção na China (Wang, 2020), e desde então o mundo começou a experienciar uma nova realidade advinda da pandemia da COVID19. O novo coronavírus nominado como Severe Acute Respiratory Syndrome Coronavirus-2 (Sars-Cov-2), e como Coronavirus Disease-19 (COVID-19) para a doença.

Inicialmente a Organização Mundial da Saúde (OMS, 2020) destacou que o grupo de idosos que são dependentes, com baixa imunidade e comorbidades associadas, se trata do grupo de maior risco para desenvolver quadros respiratórios graves capazes de levar a óbito. Além dos idosos (idade igual ou superior a 60 anos), pessoas com doenças cardíacas, pessoas com doenças pulmonares, pessoas com doenças renais ou em diálise, diabéticos, gestantes de alto risco, obesos, pessoas com 
doenças do fígado e pessoas com problemas de baixa imunidade também eram tidas como grupos que possuíam maior chance de desenvolver as formas mais graves da doença COVID-19. Contudo, em 2021, com o reconhecimento de mais variantes do vírus e o surgimento de novas ondas da doença, cada vez mais são registrados casos com quadros graves entre pessoas jovens e saudáveis, reinfecções e crescimento no número de casos e óbitos. O número de óbitos teve uma significativa diminuição após o plano de vacinação, iniciado no início de 2021 e que segue em execução, estimando-se assim que a diminuição siga sendo contínua.

O Coronavírus age, usualmente, penetrando as mucosas do nariz, boca e olhos, afetando as vias respiratórias, sendo assim algumas formas de contágio são: gotículas de saliva, tosse, espirro, aperto de mãos e objetos contaminados. Desta forma, além dos cuidados com a higiene e medidas de proteção, o distanciamento social tem sido aplicado nos países, esse representa a diminuição da interação entre as pessoas para diminuir a velocidade do contágio (Silva, 2020).

Dentre as medidas adotadas pelos governos, em nível mundial, para o enfrentamento da pandemia, encontra-se o distanciamento social e o isolamento social, em que o distanciamento remete-se a evitar o contato próximo entre as pessoas, enquanto o isolamento social acontece quando o número de contágios de dada patologia ultrapassa os valores previstos em determinado local e tempo (Silva, et al, 2020). Essas e outras medidas, como o fechamento do comércio, de instituições de ensino, realização de lockdown nas cidades, foram adotadas pela maioria da população mundial com o intuito de diminuir o contágio da população e evitar a sobrecarga dos sistemas de saúde. Todavia, a manutenção de bem-estar físico e psicológico, além de estilos de vida saudáveis são importantes para manutenção e qualidade de vida (Maria et al., 2020)

Contudo, estudos apontam que o distanciamento social pode provocar problemas de saúde mental que debilitam ainda mais o bem-estar dos idosos, bem como sentimento de solidão, insônia, ansiedade, perda de apetite e depressão (Choi, Irwin, \& Cho, 2015; Santini, et al., 2020). O isolamento social, acaba por limitar e afetar de maneira negativa a qualidade de vida dos idosos, prejudicando a saúde mental e física, ocasionando o aumento de casos de depressão, ansiedade e doenças crônicas, bem como, o crescimento no risco de doenças cardiovasculares, autoimunes, problemas neurológicos e cognitivos (Morrow-Howell, Galucia \& Swinford, 2020; Gerst-Emerson \& Jayawardhana, 2015).

O distanciamento social, bem como, a possibilidade de se enfermar, pode acarretar novos sofrimentos para as pessoas, como a depressão, em especial para os idosos. Logo, diante os diversos temas na área da saúde e da realidade vivida pelos idosos neste novo contexto de pandemia, este estudo tem como objetivo: avaliar a sintomatologia depressiva de idosos em tempo de pandemia da COVID-19.

\section{Metodologia}

Esta pesquisa se caracteriza como sendo transversal, descritiva e exploratória, com abordagem quantitativa. A coleta de dados foi realizada de forma online por meio de um formulário, que foi disponibilizado através de redes sociais, durante os meses de junho e julho de 2021. Participaram, de forma não probabilística por conveniência, 44 pessoas idosas com idades variando de 60 a 87 anos $(\mathrm{M}=62,04 ; \mathrm{DP}=6,35)$, a maioria do sexo feminino $(72,7 \%)$. Para a inclusão dos participantes na amostra, foram adotados os seguintes critérios: idade igual ou superior a 60 anos de idade e que aceitem participar da pesquisa, voluntariamente. Como critérios de exclusão: menores de 60 anos de idade.

Para a coleta de dados foram utilizados os seguintes instrumentos: Questionário sociodemográfico e clínico e a Escala de Depressão Geriátrica - EDG. O Questionário sociodemográfico e clínico foi dividido em duas partes. A primeira tinha por propósito caracterizar os participantes por meio de informações como: idade, grau de escolaridade, renda familiar, ocupação, situação conjugal, local de residência, nível de religiosidade. A segunda parte investigou os dados clínicos, como: diagnósticos de enfermidades, dentre elas a COVID-19, bem como, dados referentes à vacinação contra a COVID-19. 
A Escala de Depressão Geriátrica - EDG, foi desenvolvida por Yesavage em 1983 (Sheikh \& Yesavage, 1986). Tratase de um dos instrumentos mais comumente aplicados para rastreamento de depressão entre a população idosa (Ribeiro, Pietrobon, Rockembach, Ratzke, \& Costa, 1994). A escala é constituída por questões fáceis e possui como possibilidades de resposta: sim ou não. A princípio continha 30 perguntas, posteriormente, fundamentada na escala original, foi elaborada uma versão resumida com 15 questões. Almeida e Almeida (1999) sugerem escore de corte $\geq 5$ para determinar a presença de sintomas depressivos nos idosos. A escala tem uma variação de zero (ausência de sintomas depressivos) a quinze pontos (pontuação máxima de sintomas depressivos). Os itens descrevem sintomas específicos da depressão, que devem ser pontuados de acordo com a concordância do indivíduo com cada afirmação.

O estudo seguiu critérios recomendados pela Resolução no 465/2012 e a Resolução $N^{\circ}$ 510/2016 do Conselho Nacional de Saúde (CNS), que regulamentam pesquisas envolvendo seres humanos (Brasil, 2012; Brasil, 2016). A realização da pesquisa foi aprovada pelo do Comitê de Ética em Pesquisa da Universidade Estadual da Paraíba, sob número CAAE: 46194121.9.0000.5187. Durante todo o processo, foi assegurado aos participantes sigilo de suas identidades, além do direito de desistência de participação no estudo em qualquer momento da pesquisa.

Em relação a análise de dados, os dados sociodemográficos e clínicos serão analisados através da estatística descritiva, com o uso de medidas de posição (média e mediana) e de variabilidade (desvio padrão e amplitude). A escala foi analisada seguindo o seu manual de correção. Em seguida, foi elaborado um banco de dados com prévia codificação das respostas, usando o software Statistical Package for Social Science (SPSS) for Windows, versão 20 e diversos pacotes do programa R 3.5.2. O gráfico estatístico foi usado para representar a frequência das variáveis qualitativas em que as dimensões é proporcional à magnitude a ser representada $\left(\mathrm{n}_{i}\right.$ ou $\left.\mathrm{f}_{\mathrm{i}}\right)$. Uma outra medida muito usada para descrever dados quantitativos é a frequência acumulada, que indica quantos elementos, ou que porcentagem deles, estão abaixo de certo valor (Morettin \& Bussab, 2002).

\section{Resultados e Discussão}

Com base na análise dos dados, o perfil sociodemográfico dos participantes foi caracterizado, em sua maioria, por pessoas idosas do sexo feminino e que se declararam católicas. Ademais, a maioria dos participantes eram nordestinos, aposentados, casados, com 3 filhos, possuem baixo nível de escolaridade e renda mensal familiar entre 1 e 2 salários mínimos. Estes e outros dados sociodemográficos podem ser observado na Tabela 1.

Tabela 1. Frequências e percentuais referentes aos dados sociodemográficos.

\begin{tabular}{cc}
\hline Variáveis & $\mathbf{f}(\mathbf{\%})$ \\
Gênero & \\
Feminino & $\mathbf{3 2}(\mathbf{7 2 , 7 )}$ \\
Masculino & $12(27,3)$ \\
Faixa Etária & \\
$60-70$ anos & $\mathbf{3 2}(\mathbf{7 2 , 7 )}$ \\
$71-80$ anos & $9(20,5)$ \\
$81-87$ anos & $3(6,8)$ \\
Região do país & \\
Nordeste & $\mathbf{3 4}(\mathbf{7 7 , 2})$ \\
Sudeste & $9(20,5)$ \\
Norte & $1(2,3)$ \\
Escolaridade & \\
Sem escolaridade & $5(11,4)$
\end{tabular}




\begin{tabular}{|c|c|}
\hline $\begin{array}{l}\text { Fundamental } \\
\text { Médio } \\
\text { Superior }\end{array}$ & $\begin{array}{l}17(\mathbf{3 8 , 6 )} \\
12(27,3) \\
10(22,7)\end{array}$ \\
\hline $\begin{array}{c}\text { Condição Matrimonial } \\
\text { Solteiro(a) } \\
\text { Casado(a) } \\
\text { Divorciado(a) } \\
\text { Viúvo(a) }\end{array}$ & $\begin{aligned} 2 & (4,5) \\
30 & (\mathbf{6 8 , 2}) \\
2 & (4,5) \\
10 & (22,7)\end{aligned}$ \\
\hline $\begin{array}{l}\text { Renda Mensal* } \\
\text { Menos de } 1 \text { salário mínimo } \\
\text { Entre } 1 \text { e } 2 \text { salários mínimos } \\
\text { Entre } 2 \text { e } 5 \text { salários mínimos } \\
\text { Acima de } 5 \text { salários mínimos } \\
\text { Não tem renda } \\
\text { Não informou }\end{array}$ & $\begin{array}{c}4(9,1) \\
\mathbf{2 0}(\mathbf{4 5 , 4 )} \\
9(20,5) \\
4(9,1) \\
6(13,6) \\
1(2,3)\end{array}$ \\
\hline $\begin{array}{c}\text { Condição Laboral } \\
\text { Professor(a) } \\
\text { Aposentado(a) } \\
\text { Pescador } \\
\text { Do lar } \\
\text { Costureira } \\
\text { Auxiliar de lavanderia } \\
\text { Pensionista } \\
\text { Eletricista } \\
\text { Mecânico } \\
\text { Psicólogo(a) } \\
\text { Autônoma }\end{array}$ & $\begin{array}{c}1(2,3) \\
\mathbf{2 8}(\mathbf{6 3 , 6 )} \\
1(2,3) \\
7(15,7) \\
1(2,3) \\
1(2,3) \\
1(2,3) \\
1(2,3) \\
1(2,3) \\
1(2,3) \\
1(2,3)\end{array}$ \\
\hline $\begin{array}{c}\text { Religião } \\
\text { Católico (a) } \\
\text { Evangélico (a) } \\
\text { Espírita } \\
\text { Católica e Espírita } \\
\text { Messiânica }\end{array}$ & $\begin{array}{c}28(63,6) \\
11(25) \\
3(6,8) \\
1(2,3) \\
1(2,3)\end{array}$ \\
\hline
\end{tabular}

O envelhecimento da população é também associado ao maior percentual de mulheres em quase todas as populações mundiais, sendo denominado de feminização da velhice, e se dá pela expectativa de vida ao nascer das mulheres ser de, em média, 7,2 anos a mais que a dos homens, podendo chegar a uma expectativa de 78,8 anos (Oliveira-Filho \& Souza, 2016). Os resultados deste estudo evidenciam a feminização da velhice e a maior expectativa de vida entre as mulheres do Brasil, isto pode ser justificado, relativamente, pela maior cobertura e incentivos governamentais em políticas públicas de saúde no âmbito materno-infantil, ginecológico e obstétrico; ademais do aumento da busca das mulheres por cuidados preventivos em saúde ao decorrer da vida (Silva et al., 2018).

O alto número de idosos que se autodeclararam católicos $(63,6 \%)$ têm relação com a própria história do país, que em diferentes momentos teve influência da religião católica (Souza, 2019). No que diz respeito à condição matrimonial observouse que a maior parte da amostra, 68,2\% dos idosos são casados, assim como $22,7 \%$ são viúvos, este resultado corrobora o estudo realizado por Sousa et al. (2018) com 130 idosos, onde identificou-se um maior número de idosos casados (47,7\%), seguidos por um percentual de $32,3 \%$ de viúvos.

Em relação à condição laboral 63,6\% dos participantes estão aposentados, corroborando com a pesquisa de Annes, Mendonça, Lima, Lima e Aquino (2017) em que teve como resultado que 79,2\% das idosas participantes tinham como 
principal fonte de renda aposentada/pensionista. Quanto a Renda Mensal familiar a maioria dos idosos $(45,4 \%)$ apresentaram renda entre 1 e 2 salários mínimos, enquanto no estudo recentemente realizado por Romero e Silva (2021), que investigaram os idosos no contexto da pandemia da COVID-19 no Brasil em relação aos efeitos na renda, no trabalho e na saúde, obtiveram que a renda familiar foi menor que um salário mínimo para 31,9\% dos idosos participante.

No que diz respeito à escolaridade dos participantes desta pesquisa, obtivemos que: $38,6 \%$ possuem Ensino Fundamental, 27,3\% possuem Ensino Médio, 22,7\% possuem Ensino Superior e apenas 11,4\% não possuem escolaridade. Este resultado corrobora com Araújo et al. (2019) que realizaram um estudo com 100 idosos, e destes, 47\% afirmou ter estudado apenas até o ensino fundamental. Compreende-se assim, que o perfil sociodemográfico dos idosos deste estudo indica vulnerabilidade social, como baixa renda e baixa escolaridade. Geralmente, escolaridade e renda estão vinculados à maior suscetibilidade ao adoecimento (Visser et al., 2018), visto que têm ligação com o acesso à informação e manutenção de condições necessárias para a qualidade de vida e saúde, como trabalho, moradia, alimentação, entre outros. A escolaridade representa um importante indicador socioeconômico com impacto na saúde dos idosos, estando associada à maior ocorrência de multimorbidade (Pathirana \& Jackson, 2018) e, recentemente, isolamento social.

Resultados relativos aos dados clínicos, como doenças, ser recentemente diagnosticado com COVID-19 ou não, assim como, a vacinação contra a COVID-19 são apresentados na Tabela 2.

Tabela 2. Frequências e percentuais referentes aos dados clínicos relativos à COVID-19.

\begin{tabular}{cc} 
Variáveis & $\mathbf{f}(\boldsymbol{\%})$ \\
Enfermidade & \\
Nenhuma & $10(22,7)$ \\
Uma ou mais enfermidades & $\mathbf{3 4}(\mathbf{7 7 , 3})$ \\
Diagnóstico de COVID-19 & \\
Sim & \\
Não & $9(20,5)$ \\
& $\mathbf{3 5}(\mathbf{7 9 , 5})$ \\
Vacinação contra a COVID-19 & \\
Sim & $\mathbf{4 4}(\mathbf{1 0 0})$ \\
Não & $0(0)$ \\
Segurança psicológica após a vacinação & \\
Sim & $19(43,2)$ \\
Não & $\mathbf{2 5}(\mathbf{5 6 , 8})$ \\
\hline
\end{tabular}

Fonte: Autores (2021).

Sobre o questionamento de possuir ou não algum tipo de doença, no presente estudo, 22,7\% dos idosos entrevistados afirmaram não possuir qualquer tipo de enfermidade, contudo 77,3\% afirmaram possuir pelo menos uma enfermidade. Em que $52,3 \%$ dos idosos declararam possuir hipertensão arterial, resposta similar ao que foi identificado na pesquisa realizada por Araújo et al., (2017), onde a maioria (58\%) da amostra declarou ter hipertensão. Romero e Silva (2021) identificaram que, no contexto pandêmico da COVID-19, a hipertensão é a Doença Crônica Não Transmissível (DCNT) com a maior prevalência entre as pessoas idosas $(43,8 \%)$ e que mais de 58\% dos idosos têm pelo menos uma DCNT de risco para COVID-19 grave.

Em relação ao diagnóstico de COVID-19, observou-se que 79,5\% da amostra não foi diagnosticada com a enfermidade recentemente. Já em relação a vacinação, todos os idosos afirmaram já terem sido vacinados, contudo, quando perguntados se a vacinação gerou algum tipo de segurança psicológica e/ou mudou seu comportamento social, a maioria dos participantes afirmou que não $(56,8 \%)$. A população idosa tem sido viabilizada e priorizada em vários países durante a 
vacinação contra a COVID-19, tendo em vista a redução da mortalidade e a necessidade de dar visibilidade às particularidades do cuidado às pessoas idosas. Logo, a vacinação tem sido compreendida em todo o mundo como uma estratégia primordial de promoção e proteção da saúde dos idosos (Souto \& Kabad, 2020).

No tocante aos resultados da Escala de Depressão Geriátrica (EDG-15), estes revelaram que não há presença de depressão em 75\% dos idosos estudados, observa-se também que 13,6\% apresentaram depressão moderada e apenas 11,4\% têm sintomas de depressão severa (Figura 1). Estes resultados corroboram com os resultados encontrados por Abrantes et al. (2019), em seu estudo sobre sintomas depressivos em idosos na atenção básica à saúde também utilizando a EDG, em que $75,0 \%$ dos idosos foram classificados como "sem sintomas depressivos".

Figura 1. Frequência acumulada de casos de depressão entre os

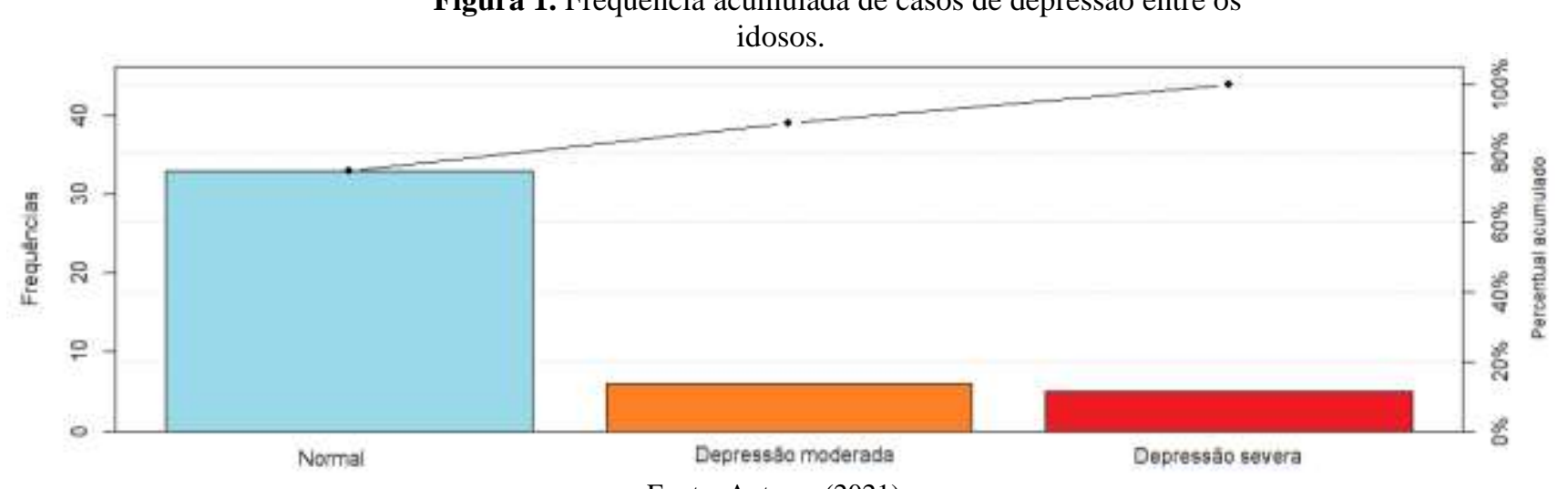

Fonte: Autores (2021).

Ao encontro dos resultados obtidos neste estudo, a pesquisa de Pereira-Ávila et al. (2021), que teve como objetivo identificar os fatores associados aos sintomas de depressão entre idosos durante a pandemia do COVID-19, obteve como resultado que a maioria $(91,9 \%)$ dos idosos não apresentou sintomas de depressão ou sintomas mínimos, seguidos de 5,7\% que apresentaram sintomas moderados, 2,0\% sintomas moderadamente graves e 1,4\% sintomas de depressão severa.

Levando em consideração que $25 \%$ dos idosos apresentaram sintomatologia depressiva moderada ou severa, entendese que o medo da contaminação e o isolamento social imposto pelas medidas protetivas para conter o vírus, impulsionou o aparecimento de alterações na saúde mental, principalmente de adultos idosos (Lima et al., 2020). Smith, Haedtke and Shibley (2015) apontam que a população idosa requer atenção, dado o aumento no número de idosos que manifestaram fatores de risco para a depressão, portanto, considera-se fundamental estudos e ferramentas de rastreio e detecção da depressão, visando tratamento e prevenção. O quadro depressivo nos idosos, se diferencia de outras idades em razão das distinções de sintomatologia e dos contextos de vida característicos da idade, mostrando uma redução da resposta emocional, falta de afeto e hegemonia de sintomas, perda de prazer nas atividades cotidianas, perda de energia, dentre outros.

Levando em consideração que $25 \%$ dos idosos apresentaram sintomatologia depressiva moderada ou severa, entendese que o medo da contaminação e o isolamento social imposto pelas medidas protetivas para conter o vírus, impulsionou o aparecimento de alterações na saúde mental, principalmente de adultos idosos (Lima et al., 2020). Smith, Haedtke and Shibley (2015) apontam que a população idosa requer atenção, dado o aumento no número de idosos que manifestaram fatores de risco para a depressão, portanto, considera-se fundamental estudos e ferramentas de rastreio e detecção da depressão, visando tratamento e prevenção. O quadro depressivo nos idosos, se diferencia de outras idades em razão das distinções de sintomatologia e dos contextos de vida característicos da idade, mostrando uma redução da resposta emocional, falta de afeto e hegemonia de sintomas, perda de prazer nas atividades cotidianas, perda de energia, dentre outros. 
De acordo com o estudo de Gonçalves, et al. (2020), a sintomatologia depressiva é uma variável preditora de sintomas somáticos, sendo então importante entender que na avaliação e compreensão das queixas somáticas de idosos, se considerarem sintomas psicológicos, nomeadamente, a sintomatologia depressiva. Sintomas depressivos podem ser mascarados ou potencializados pelo envelhecimento, doenças associadas e uso de medicações. Diante de sintomas que caracterizam um quadro depressivo na pessoa idosa pode prejudicar sua qualidade de vida, afetar sua autonomia e independência, afetividade e sua funcionalidade mental e física. Nesse sentido, é fundamental o monitoramento da saúde mental dos idosos dado que, principalmente durante situações de pandemia, à exemplo a pandemia da COVID-19, algumas pessoas idosas apresentam dificuldades resultantes do distanciamento e/ou isolamento social, pela instabilidade dos vínculos afetivos, o que pode provocar tristeza, angústia e solidão, podendo serem levados à quadros depressivos.

No que diz respeito aos dados referentes aos itens da EDG desta pesquisa serão observados na Tabela 3. Percebe-se que os idosos em sua maioria estavam satisfeitos com a sua vida $(84,1 \%)$ e se sentiam felizes na maioria das vezes $(84,1 \%)$, estando de bom humor na maioria das vezes (70,5\%), bem como, pensam que é maravilhoso estar vivo (93,2\%). Estes dados corroboram com a pesquisa realizada por Abrantes et al. (2019) que obteve como resultado que 84,2\% dos idosos afirmaram estar satisfeitos com sua vida, $76,1 \%$ se sentiam felizes, e que 74,6\% sentiam-se de bom humor a maior parte do tempo, mostrando o grau de importância da felicidade como um indicativo de bem-estar, podendo esse ser tido como um fator de proteção contra os sintomas depressivos.

Tabela 3. Percentual dos níveis relacionados aos 15 itens da Escala de Depressão Geriátrica.

\begin{tabular}{|c|c|c|}
\hline Variáveis & $\begin{array}{l}\text { Sim } \\
\mathbf{f}(\%)\end{array}$ & $\begin{array}{l}\text { Não } \\
\text { f (\%) }\end{array}$ \\
\hline 1- Você está satisfeito com a sua vida? & $37(84,1)$ & $7(15,9)$ \\
\hline $\begin{array}{l}\text { 2- Você deixou de lado muitas de suas } \\
\text { atividades e interesses? }\end{array}$ & $31(70,5)$ & $13(29,5)$ \\
\hline 3- Você sente que sua vida está vazia? & $13(29,5)$ & $31(70,5)$ \\
\hline 4- Você sente-se aborrecido com frequência? & $20(45,5)$ & $24(54,5)$ \\
\hline $\begin{array}{l}\text { 5- Está você de bom humor na maioria das } \\
\text { vezes? }\end{array}$ & $31(70,5)$ & $13(29,5)$ \\
\hline 6- Você teme que algo de ruim lhe aconteça? & $20(45,5)$ & $24(54,5)$ \\
\hline 7- Você se sente feliz na maioria das vezes? & $37(84,1)$ & $7(15,9)$ \\
\hline 8- Você se sente frequentemente desamparado? & $9(20,5)$ & $35(79,5)$ \\
\hline $\begin{array}{l}\text { 9- Você prefere permanecer em casa do que } \\
\text { sair e fazer coisas novas? }\end{array}$ & $31(70,5)$ & $13(29,5)$ \\
\hline $\begin{array}{l}\text { 10- Você sente que tem mais problemas de } \\
\text { memória do que antes? }\end{array}$ & $22(50)$ & $22(50)$ \\
\hline 11- Você pensa que é maravilhoso estar vivo? & $41(93,2)$ & $3(6,8)$ \\
\hline 12- Você se sente inútil? & $10(22,7)$ & $34(77,3)$ \\
\hline
\end{tabular}




\begin{tabular}{ccc} 
13- Você se sente cheio de energia? & $26(59,1)$ & $18(40,9)$ \\
$\begin{array}{c}\text { 14- Você sente que sua situação é sem } \\
\text { esperança? }\end{array}$ & $6(13,6)$ & $38(86,4)$ \\
$\begin{array}{c}\text { 15- Você pensa que a maioria das pessoas estão } \\
\text { melhores do que você? }\end{array}$ & $7(15,9)$ & $37(84,1)$ \\
\hline
\end{tabular}

Fonte: Autores (2021).

Alguns idosos desta pesquisa relataram preocupação com problemas de memória (50\%) e que preferem permanecer em casa do que sair e fazer coisas novas (70,5\%), resultados semelhantes foram encontrados no estudo de Abrantes et al. (2019). Sendo assim, pesquisas acerca dos contextos que as pessoas idosas estão introduzidas, modo de vida e comportamentos que promovem saúde, são essenciais para a promoção de um cuidado integral dessas pessoas (Abrantes et al., 2019), compreendendo como sujeitos biopsicossociais.

Portanto, são importantes estratégias e ações de cuidado com os idosos, que atente-se à prestação de acolhimento psicológico, seguridade na transferência de informações, com a finalidade de não provocar mais angústia aos idosos, viabilização de canais de escuta para propiciar resolução de problemas na rotina de isolamento e, sobretudo, fortalecimento da rede de apoio familiar (Alves \& Magalhães, 2020).

\section{Considerações Finais}

O estudo procurou avaliar a sintomatologia depressiva de idosos em tempo de pandemia da COVID-19. A partir dos achados, verificou-se que na maioria dos idosos não há presença de depressão, observa-se que os idosos em sua maioria estavam satisfeitos com a sua vida, se sentiam felizes e de bom humor na maioria das vezes, assim como, pensavam que é maravilhoso estar vivo.

Constatou-se também que uma quantidade significativa dos idosos pesquisados apresentaram sintomatologia depressiva moderada ou severa. Diante disso, é possível compreender que o isolamento e/ou distanciamento social, embora necessário durante a pandemia da COVID-19, pode ocasionar efeitos prejudiciais à saúde mental da pessoa idosa.

Considera-se, no entanto, que este estudo apresenta limitações, na medida em que o número de participantes foi reduzido. Neste sentido, sugerem-se estudos futuros sobre a saúde mental de pessoas idosas, visto que a vivência da pandemia da COVID-19 acarretou e segue acarretando consequências socioeconômicas, psicológicas e fisiológicas para a população em geral. Mais que isso, deve-se planejar e executar ações em saúde e estratégias de promoção de saúde, investindo em projetos voltados à educação, segurança, alimentação e na rede de serviços de saúde, especialmente da saúde mental, como serviços psicológicos.

\section{Referências}

Abrantes, G. G., Souza, G. G., Hélder, N. M. C., Rocha, N. B., et al. (2019). Sintomas depressivos em idosos na atenção básica à saúde: Depressive symptoms in older adults in basic health care. Revista Brasileira de Geriatria e Gerontologia, Rio de Janeiro, v. 22, n. 4, p. 1-7, 2019. FapUNIFESP (SciELO). https://www.scielo.br/pdf/rbgg/v22n4/pt_1809-9823-rbgg-22-04-e190023.pdf.

Almeida O. P., \& Almeida S.A. (1999). Confiabilidade da versão brasileira da Escala de Depressão Geriátrica (GDS) versão reduzida. Arquivos de NeuroPsiquiatria, 57(2)B:421426.

Alves, J. do N., \& Magalhães, I. M. de O. (2020). Implicações na saúde mental de idosos diante do contexto pandêmico da COVID-19. Revista enfermagem atual. (Ed. Especial).

Annes, L. M., Mendonça, H. G., Lima, F. M., Lima, M. A., \& Aquino, J. M. (2017). Perfil sociodemográfico e de saúde de idosas que participam de grupos de terceira idade em Recife, Pernambuco. Rev Cuid. 2017; 8(1): 1499-508. http://dx.doi.org/10.15649/cuidarte.v8i1.365 
Araújo, I. C. D. et al. (2019). Perfil sociodemográfico e qualidade de vida de idosos de um centro de referência do idoso do oeste paulista. São Paulo: Colloquium Vitae, vol. 11, n. 1, p. 17-23, abr., 2019.

Brasil. (2012). Resolução $n^{\circ} 466$, de 12 de dezembro de 2012. Dispõe sobre diretrizes e normas regulamentadoras de pesquisas envolvendo seres humanos. Diário Oficial [da] República Federativa do Brasil, Brasília, DF, 13 jun. 2013. http://bit.ly/1mTMIS3.

Brasil. (2016). Resolução $n^{\circ} 510$, de 07 de abril de 2016. Dispõe sobre as normas aplicáveis a pesquisas em Ciências Humanas e Sociais. Diário Oficial [da] República Federativa do Brasil, Brasília, DF, 24 maio 2016. http://bit.ly/2fmnKeD.

Chaimowicz, F. (2013). Transição Demográfica. In: F. Chaimowicz, Saúde do Idoso, (2ª ed.), 27-31. Belo Horizonte: NESCON UFMG.

Choi, H., Irwin, M. R., \& Cho, H. J. (2015). Impact of social isolation on behavioral health in elderly: Systematic review. World J Psychiatry; 5(4):432-438.

Coelho, F. G.de M., Gobbi, S., Costa, J. L. R., \& Gobbi, L. T. B. (2013). Exercício físico no envelhecimento saudável e patológico: da teoria à prática. Curitiba (PR): CRV

Fortes, T. F. R., Portuguez, M. W., \& Argimon, I. I. L. (2009). A resiliência em idosos e sua relação com variáveis sociodemográficas e funções cognitivas. Estudos de Psicologia (Campinas), 26(4), 455-463. https://dx.doi.org/10.1590/S0103-166X2009000400006

Garcia, M. A. A., Rodrigues, M. G., \& dos Santos Borega, R. (2012). O envelhecimento e a saúde. Rev Ciênc Méd.11(3).

Gerst-Emerson, K., \& Jayawardhana, J. (2015). Loneliness as a public health issue: the impact of loneliness on health care utilization among older adults. Am J Public Health 2015; 105(5):1013-1019.

Gonçalves, D., Afonso, R., Dias, I., Lopes, T., Pereira, H., Esgalhado, M., Monteiro S., \& Loureiro, M. (2020). Sintomas somáticos, sintomatologia depressiva e ansiógena em pessoas idosas. Psicologia, saúde \& doenças. 020, 21(1), 131-136 ISSN - 2182-8407 Sociedade Portuguesa de Psicologia da Saúde - SPPS www.sp-ps.pt DOI: http://dx.doi.org/10.15309/20psd21012.

Instituto Brasileiro de Geografia e Estatística - IBGE. (2018). Número de idosos cresce $18 \%$ em 5 anos e ultrapassa 30 milhões em 2017. https://agenciadenoticias.ibge.gov.br/agencia-noticias/2012-agencia-denoticias/noticias/20980-numero-de-idosos-cresce-18-em-5-anos-e-ultrapassa-30milhoes-em2017.

IBGE (2019, fevereiro). Retratos: a revista do IBGE. Rio de Janeiro: IBGE, CCS.

Lima, R. C. (2020). Distanciamento e isolamento sociais pela Covid-19 no Brasil: impactos na saúde mental. Physis: Revista de Saúde Coletiva, Rio de Janeiro, v. 30(2), e300214.

Maria, M., Ferro, F., Ausili, D., Alvaro, R., De Marinis, M. G., Di Mauro, S., Matarese, M., \& Vellone, E. (2020). Development and Psychometric Testing of the Self-Care in COVID-19 (SCOVID) Scale, an Instrument for Measuring Self-Care in the COVID-19 Pandemic. International journal of environmental research and public health, 17(21), 7834. https://doi.org/10.3390/ijerph17217834.

Morettin, P. A., \& Bussad, W. O. (2002). Estatística Básica. 5. ed. São Paulo: Saraiva.

Morrow-Howell, N., Galucia, N., \& Swinford, E. (2020). Recovering from the COVID-19 Pandemic: A Focus on Older Adults. Journal of Aging \& Social Policy, 32: 4-5, 526-535, https://doi.org/10.1080/08959420.2020.1759758.

Oliveira-Filho, E. C.; Souza, L. G. de S. C. N. de. (2017). Causas e consequências da redução da taxa de fecundidade no Brasil. 2016. Trabalho de Conclusão de Curso - Faculdade de Enfermagem do Centro Universitário de Brasília, Brasília, .

Organização Mundial da Saúde (2020). Declarou a Pandemia de Coronavírus em 11 de março de 2020. https://www.bbc.com/portuguese/geral51842518.

Pathirana, T. I., Jackson, C. A. (2018). Socioeconomic status and multimorbidity: a systematic review and meta-analysis. Aust N Z J Public Health; $42: 186-94$.

Pereira-Ávila, F.M.V., Lam, S.C., Goulart, M.C.L., Góes, F.G.B., Pereira-Caldeira, N.M.V., \& Gir, E. (2021). Fatores associados aos sintomas de depressão entre idosos durante a pandemia da COVID-19. enferm. 30 • 2021 • https://doi.org/10.1590/1980-265X-TCE-2020-0380.

Ribeiro, M. A. M., Pietrobon, R. S., Rockembach, R. A., Ratzke O. \& Costa, P. A. B. (1994). Prevalência da depressão em idosos institucionalizados em tempo integral. Rev Psiquiatr Clin. 21(1):4-8.

Romero, D. E., \& Silva, D. R. P. (2021). Idosos no contexto da pandemia da COVID-19 no Brasil: efeitos nas condições de saúde, renda e trabalho. https://doi.org/10.1590/0102-311X00216620.

Sheikh, J. I., \& Yesavage, J. A. (1986). Escala de Depressão Geriátrica (GDS): Evidências recentes e desenvolvimento de uma versão mais curta. Gerontologista clínico: The Journal of Aging and Mental Health, 5 (1-2), 165-173.

Silva, P. A. B., Santos, F. C., Soares, S. M., \& Silva, L. B. (2018). Perfil sociodemográfico e clínico de idosos acompanhados por equipes de Saúde da Família sob a perspectiva do gênero. Rev. pesqui. cuid. fundam. (Online), 10(1), 97-105. https://doi.org/10.9789/2175-5361.2018.v10i1.97-105.

Silva, J. da. (2020). Saúde mental de idoso no contexto da COVID-19/Josevânia da Silva - Campina Grande: EDUEPB, 2020.

Silva, M. V.S., Rodrigues, J. A., Ribas, M. S., Sousa, J. C. S., Castro, T. R. O., Santos, B. A., Sampaio, J. M. C, \& Pegoraro, V. A. (2020). O impacto do isolamento social na qualidade de vida dos idosos durante a pandemia por COVID-19. Enfermagem Brasil. 19(4Supl), 34- 4.

Smith, M., Haedtke, C., \& Shibley, D. (2015). Late life depression detection: An evidencebased guideline. Journal Gerontolical Nursing, 41(2), 18-25. https://doi.org/doi:10.3928/00989134-20150115-99. 
Research, Society and Development, v. 10, n. 14, e520101422245, 2021

(CC BY 4.0) | ISSN 2525-3409 | DOI: http://dx.doi.org/10.33448/rsd-v10i14.22245

Sousa, F. de J. D. et al. (2018) Perfil sociodemográfico e suporte social de idosos na atenção primária. Recife: Ver. enferm. UFPE online, vol. 12, n. 4, p. 824831 , abr.

Souto, E. P., \& Kabad, J. (2020). Hesitação vacinal e os desafios para enfrentamento da pandemia de COVID-19 em idosos no Brasil. Rev. bras. geriatr. gerontol. 23 (5). https://doi.org/10.1590/1981-22562020023.210032.

Souza, A. R. (2019). Pluralidade cristã e algumas questões do cenário religioso brasileiro. Revista USP, (120), 13-22. https://doi.org/doi:10.11606/issn.23169036.v0i120p13-22.

Visser, M., Wijnhoven, H. A., Comijs, H. C., Thomése, F. G., Twisk, J. W., \& Deeg, D. J. (2019). A healthy lifestyle in old age and prospective change in four domains of functioning. Journal of aging and health, 31(7), 1297-1314. https://doi.org/doi:10.1177/0898264318774430. 